\title{
The Incidence and Association of Mental Depression with Symptomatic Lumbar Degenerative Disc Disease and Treatment Outcome
}

\author{
Lukman Olalekan Ajiboye, Muhammad Oboirien \\ Usmanu Danfodiyo University Teaching Hospital, Sokoto, Nigeria \\ Email: ajiboyelo@yahoo.com, moboirien@yahoo.com
}

How to cite this paper: Ajiboye, L.O. and Oboirien, M. (2018) The Incidence and Association of Mental Depression with Symptomatic Lumbar Degenerative Disc Disease and Treatment Outcome. Health, 10, 1487-1497.

https://doi.org/10.4236/health.2018.1011114

Received: September 26, 2018

Accepted: November 12, 2018

Published: November 15, 2018

Copyright $\odot 2018$ by authors and Scientific Research Publishing Inc. This work is licensed under the Creative Commons Attribution International License (CC BY 4.0).

http://creativecommons.org/licenses/by/4.0/ Open Access

\begin{abstract}
Study Design: Prospective analytical study. Objectives: The aim was to determine the association between mental depression and symptomatic Lumbar Degenerative Disc Disease (LDDD) in patients with no previous background of mental disorder. We also aimed at determining the incidence of mental depressions in patients with LDDD and the effects of the treatment on the mental depression. Methodology: One hundred and sixty patients with no prior history of mental or behavioral disorders who presented with low back pains arising from LDDD and met inclusion criteria were studied. The clinical findings and Depression Screening Test pro-forma were completed for each. The extracted information was analyzed using Statistical Package for Social Science (SPSS) version 24.0. The statistical significance was set at $\mathrm{P}<$ 0.05. Results: One hundred and fifty-three patients completed the study, with a male to female ratio of $1: 1.5$ and the mean age of the patients was 48.5 years. The marital status was $4.4 \%$ single, $86.9 \%$ married, $3.8 \%$ divorce/separate and $5 \%$ widow/widower. Their levels of education were: no formal education (10.00\%), primary school level (8.10\%), secondary level (27.50\%) and Tertiary level (54.40\%). Conclusions: This study showed the incidence of mental depression in $32 \%$ of the patients with LDDD. We also noted a statistically significant relationship between symptomatic LDDD and level of mental depression with significant improvement in the level of depression at $6^{\text {th }}$ month after treatment. Hence, assessment of the patients' mental health is important in the management of LDDD.
\end{abstract}

\section{Keywords}

Incidence, Mental Depression, Symptomatic Lumbar Degenerative Disc Disease, Outcome, Association 


\section{Introduction}

Chronic back pains due to Lumbar Degenerative Disc Disease (LDDD) are common, with up to $90 \%$ of the people expected to experience chronic low back pain (CLBP) at one time or the other during their lifetime [1]. Globally, the most common cause of CLBP is slipped disc or LDDD, which results in spinal cord or nerve root compression. However, CLBP with morbidity and loss of normal function for daily activity is a major concern and it is reported in the literatures to be associated with psychologic disorders like mental depression, anxiety, sleeping disorders and poor physical performance [2] [3] [4]. There is paucity of information about LDDD and mental depression in our settings and incidence of mental health disorders among patients with LDDD is not known in our environment.

Lumbar DDD is a degenerative disease of lumbar inter-vertebral discs (IVDs), which consequently causes degeneration in vertebral bodies, and/or associated joints of axial spine and clinical syndromes of discogenic back pain, lumbar radiculopathy, facet joint osteoarthritis, and segmental instability [5] [6].

Mental depression also called clinical depression or major depressive disorder is a form of mood disorder which causes persistent feeling of sadness, loss of interest, affects feeling, thinking and behavior and can lead to a variety of emotional and physical problems [7] [8] [9] [10]. Although it may occur only once during a life time but typically in multiple episodes with each episode characterized with multiple of the following: sadness, tearfulness, hopelessness, anger, irritability or frustration, loss of interest or pleasure in most or all normal activities, sleep disorders, tiredness and lack of energy, eating disorders, anxiety, feelings of worthlessness or guilt, recurrent thoughts of death, suicidal thoughts, suicide attempts or suicide, and unexplained physical symptoms or its severity like back pain or headaches [7] [8] [9] [10]. Depression unfortunately often goes undiagnosed and untreated in older adults, and they may feel reluctant to seek help and symptoms of depression may be different or less obvious in adults.

Many literatures and studies have shown a strong relationship between chronic pain and depression but the extent to which depression and chronic pain are associated remains a controversial issue that empirical studies have failed to resolve completely [3] [11]. An evaluation of relevant literature provides support for the association between the two syndromes and suggests that coexisting pain and depression may be a final common presentation reached by a number of potential pathways [3] [11].

Treatment Modalities include non-operative such as pharmacological, physiotherapy or physical therapy, epidural steroid injection and different types of operative managements. Prognosis of any of the treatment depends on the extent of the pathology, neurological involvement, type of treatments, adequacy of care and psychology of the patient.

The objective of our study was to determine the association between mental depression and symptomatic Lumbar Degenerative Disc Disease in adult pa- 
tients with no background of mental or behavioral disorder. We also aimed at determining the incidence of mental depressions in patients with LDDD and the effects of the treatments on the mental depression.

\section{Methods}

One hundred and sixty adults with symptomatic LDDD were studied. All patients had follow up periods of at least six months after treatment. These participants were recruited consecutively after informed consent was taken. The inclusion criteria were patients aged 31 to 60 years with clinical features of symptomatic LDDD or its complication(s) presenting at outpatient clinic or emergency. The exclusion criteria were low back pain from causes other than LDDD, patients who have had spinal surgery for LDDD, previous spine pathology (such as injury, infection, and tumor), congenital or structural spinal deformity, and patients with history or risk of depression or other mental health/ behavioral disorders predating LDDD.

At presentation, LDDD was diagnosed by detailed clinical history taking, physical examinations, and lumbosacral imagings. All patients had initial analgesics (parenteral and/or oral) depending on the pain severity and history of co-morbidity. Other clinical investigations such as cervical, knee and hip imagings as well as blood and urine tests were done depending on individual's pathology. The socio-demographic and symptomatologic information were taken with the use of questionnaires. Also each patient's level of mental depression associated with the chronicity of LDDD was assessed by using Depression Screening Test by Ivan Goldberg questionnaires at presentation, 3 and 6 months after treatment and clinical improvement of the symptoms were assessed by Oswestry Disability Index version 2 at presentation and 6-month follow up.

The treatment modalities were lumbar posterior spine fusion with or without decompression, epidural steroid injection and medical therapy (analgesics with or without neurovitamin and muscle relaxants). The patients were divided into these three study groups based on the clinical symptomatology and informed decision of the patients. The clinical responses to the treatment were followed up. So, patients who had no satisfactory response following medical or epidural steroid injection were accordingly further treated with steroid injection or operative care respectively as clinical status deserved. The patients with persistent symptoms of mental depression after adequate treatment or severe and uncontrollable mental depression at presentation were co-managed with Mental Health Physician. The information was analyzed using Statistical Package for Social Science (SPSS) version 24.

\section{Results}

The study was conducted among one hundred and sixty patients but one hundred and fifty three of them completed the duration of follow up. There were 61 males and 92 females with male to female ratio of 1:1.5. The mean age was 45.50 
\pm 14.50 years (range $31-60$ ). The symptom duration of LDDD among the patients spanned between 1 to 14 years with the mean symptoms duration of 4.46 years. About $85.6 \%$ of the patients presented at Surgical Out-Patient Department while the rest were at emergency. Figure 1 showed the levels of education among the participants. Figure 2 showed the marital statuses of the participants. Table 1 shows the level of Mental Depression (Ivan Goldberg Score) and ODI Score (\%) at Presentation. Figure 3 showed the treatment modalities received by the participants. Figure 4 showed the relationship between the level of mental Depression Score and ODI Score (\%) at Presentation. Table 2 showed the level mental Depression Scores and ODI Scores (\%) at 6 month after treatment. Figure 5 showed the relationship between level of mental Depression Score and ODI Score (\%) at 6-month after treatment.

The figure bellow shows the level of education among studied subjects.

The figure below showed the marital statuses of the participants.

This table bellow shows the level of Mental Depression (Ivan Goldberg Score) and ODI Score (\%) at Presentation.

The figure below showed the treatment modalities received by the participants.

The figure below shows the relationship on Bar chart of the mental Depression Score and ODI Score (\%) at Presentation.

This table below showed the level mental Depression Scores and ODI Scores (\%) at 6 month after treatment.

The figure below shows the relationship (on Bar chart) of the mental Depression Score and ODI Score (\%) at $6^{\text {th }}$ month follow up after the treatments.

Table 1. Depression Screening Test (Ivan Goldberg Score) and ODI Score (\%) at Presentation.

\begin{tabular}{ccccccccc}
\hline & \multicolumn{7}{c}{ ODI Score (\%) } & \multirow{2}{*}{ Total } \\
\cline { 3 - 7 } & $0-9$ & 5 & 86 & 38 & 17 & 0 & 86 \\
& $0-20$ & $21-40$ & $41-60$ & $61-80$ & $81-100$ & \\
\hline & $10-17$ & 3 & 18 & 11 & 3 & 0 & 18 \\
Depression Screening & $18-21$ & 2 & 17 & 6 & 6 & 0 & 17 \\
Test by Ivan Goldberg & $22-35$ & 0 & 18 & 4 & 8 & 3 & 18 \\
& $36-53$ & 0 & 9 & 2 & 3 & 4 & 9 \\
Total & $>53$ & 0 & 5 & 0 & 1 & 4 & 5 \\
\hline
\end{tabular}

Key: Oswestry Disability Index (ODI) (\%): 0\% to 20\%: Minimal Disability; $21 \%$ - 40\%: Moderate Disability; 41\% - 60\%: Severe Disability; 61\% - 80\%: Crippled; $81 \%$ - 100\%: Bed/Wheel Chair Bound; Comments: One hundred and ten (71.9\%) of the participants presented with severe scores of ODI (41 - 100\%); Depression Screening Test (Ivan Goldberg Score): 0 - 9 No Depression Likely; 10 - 17 Possibly Mildly Depressed; 18 21Borderline Depression; 22 - 35 Mild-Moderate Depression; 36 - 53 Moderate-Severe Depression; $\geq 54$ Severe Depression; Comments: One hundred and twenty one of the participants (68\%) were unlikely to have depression symptoms (0 - 17 points); Forty nine (32\%) of the participants had depression symptoms with severe depression symptoms in $14(9.2 \%)$ of them. 
Table 2. 6-Month Post Op Depression Screening Test scores and ODI Score (\%).

\begin{tabular}{cccccccc}
\hline & & \multicolumn{5}{c}{ 6-Month Post Op ODI Score (\%) } & \multirow{2}{*}{ Total } \\
\cline { 3 - 6 } & & $0-20$ & $21-40$ & $41-60$ & $61-80$ & $81-100$ & \\
\hline 6-Month & $10-17$ & 9 & 17 & 4 & 3 & 1 & 34 \\
Post Op & $18-21$ & 2 & 2 & 2 & 0 & 0 & 6 \\
Mental & $22-35$ & 0 & 1 & 0 & 1 & 0 & 2 \\
$\begin{array}{c}\text { Depression } \\
\text { Screening }\end{array}$ & $36-53$ & 1 & 0 & 1 & 0 & 0 & 2 \\
Score & $>53$ & 1 & 1 & 1 & 0 & 1 & 4 \\
\multicolumn{1}{c}{ Total } & & 45 & 84 & 16 & 6 & 2 & 153 \\
\hline
\end{tabular}

Key: ODI and Depression scores as in Table 1; Comments: 1) There is general improvement in the level of mental depression and ODI (24 patients had severe ODI score (41 - 100\%) at 6-month follow up); 2) One hundred and thirty nine of the participants $(90.8 \%)$ were unlikely to have depression symptoms $(0-21$ points); 3) Fourteen $(9.2 \%)$ of the participants had depression symptoms; 4 ) There is statistically significant association between level of mental depression and ODI $(P$ value $=0.001)$.

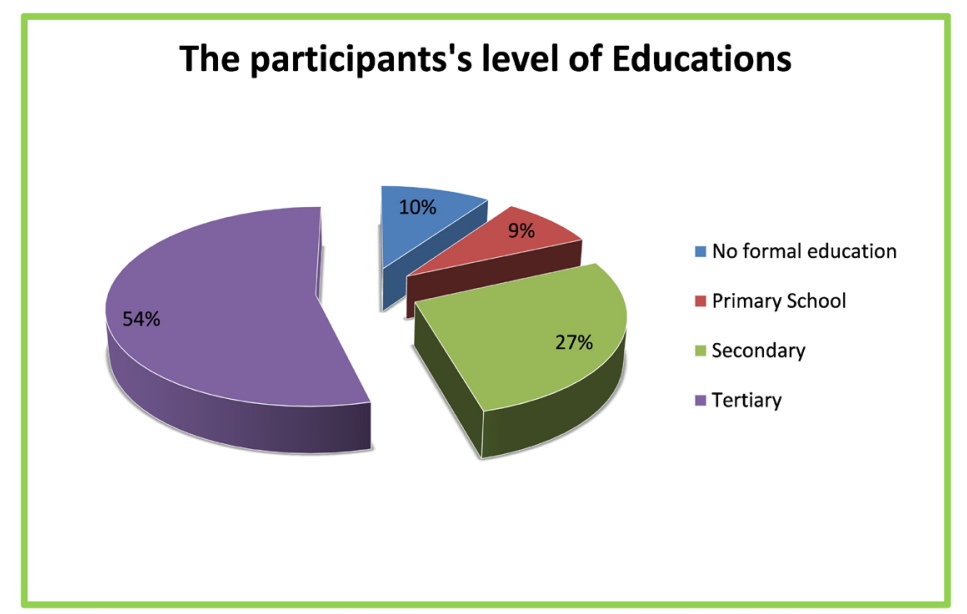

Figure 1. The level of education among the participants. Comments: $54 \%$ of the participants had tertiary level of education; $9 \%$ of the participant had no formal education.

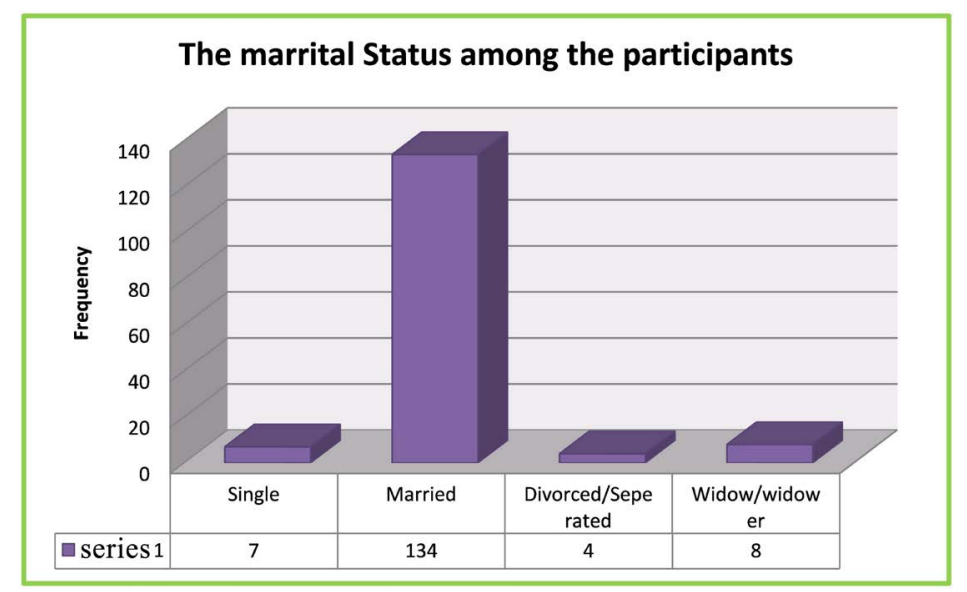

Figure 2. The bar chart showing the marital status among the participants. Comments: $134(87.6 \%)$ of the participants were married; $4(2.6 \%)$ of the participant were separated/divorced. 


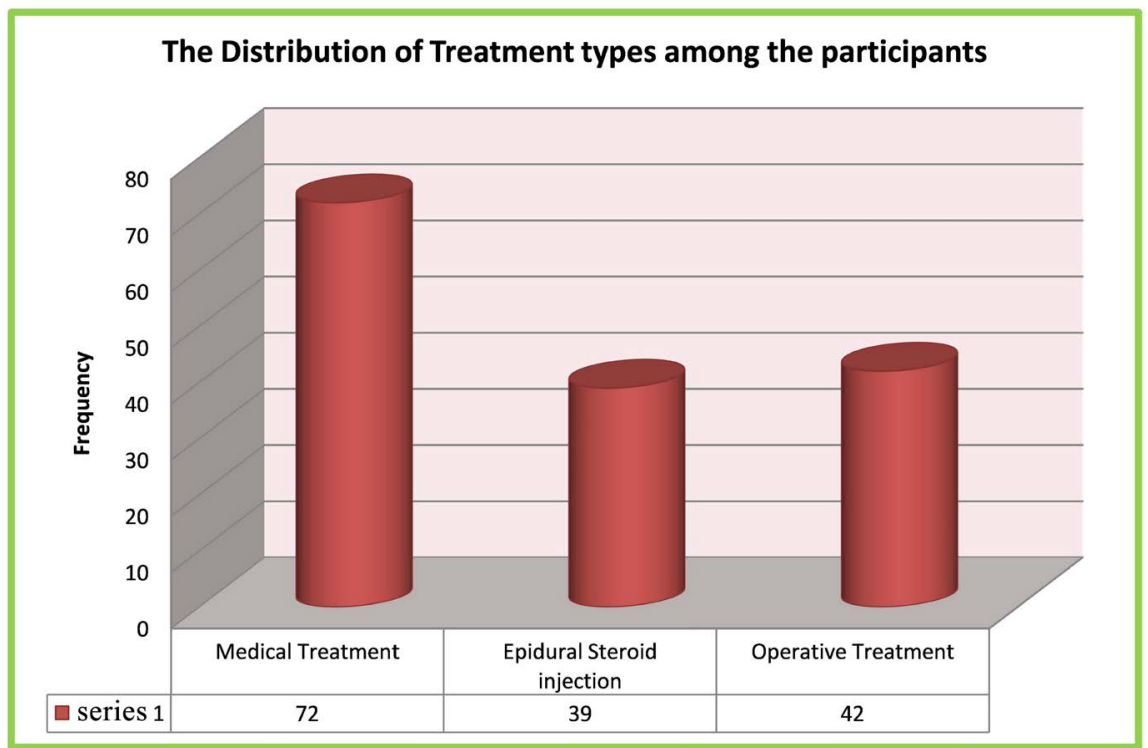

Figure 3. The bar chart showing the types of the treatment received by the participants. Comments: Seventy two $(47.1 \%)$ of the participant had mainly medical treatment which is the majority; $25.5 \%$ and $27.5 \%$ of the participant had Epidural steroid injection and operative treatments respectively.

\section{Bar Chart}

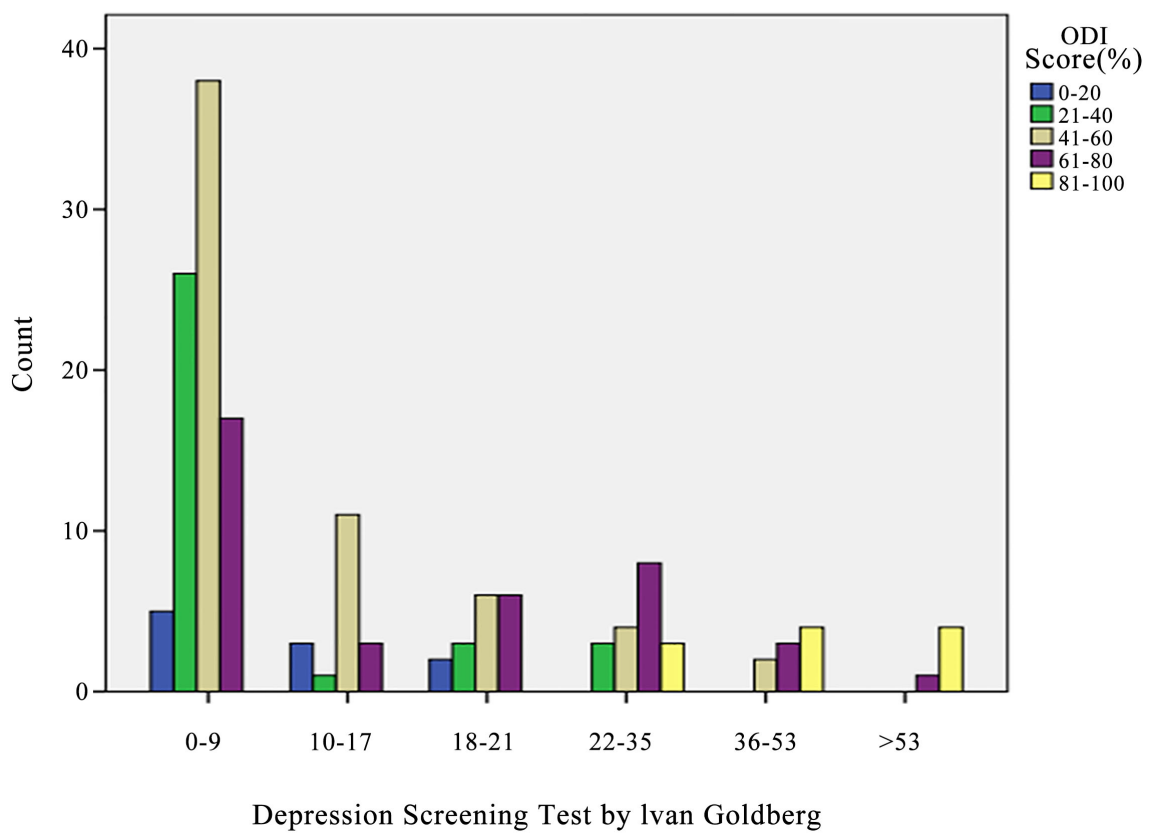

Figure 4. The relationship between the level of mental Depression Score and ODI Score (\%) at Presentation. Key: Oswestry Disability Index (ODI) (\%): 0\% to 20\%: Minimal Disability; 21\% - 40\%: Moderate Disability; 41\% - 60\%: Severe Disability; 61\% - 80\%: Crippled; 81\% - 100\%: Bed/Wheel Chair Bound; Depression Screening Test (Ivan Goldberg Score): 0 - 9: No Depression Likely; 10 - 17: Possibly Mildly Depressed; 18 - 21: Borderline Depression; 22 - 35: Mild-Moderate Depression; 36 - 53: Moderate-Severe Depression; $\geq 54$ : Severe Depression. Comments: The more severe the ODI score is, the more is the severity of mental Depression among the participants. 


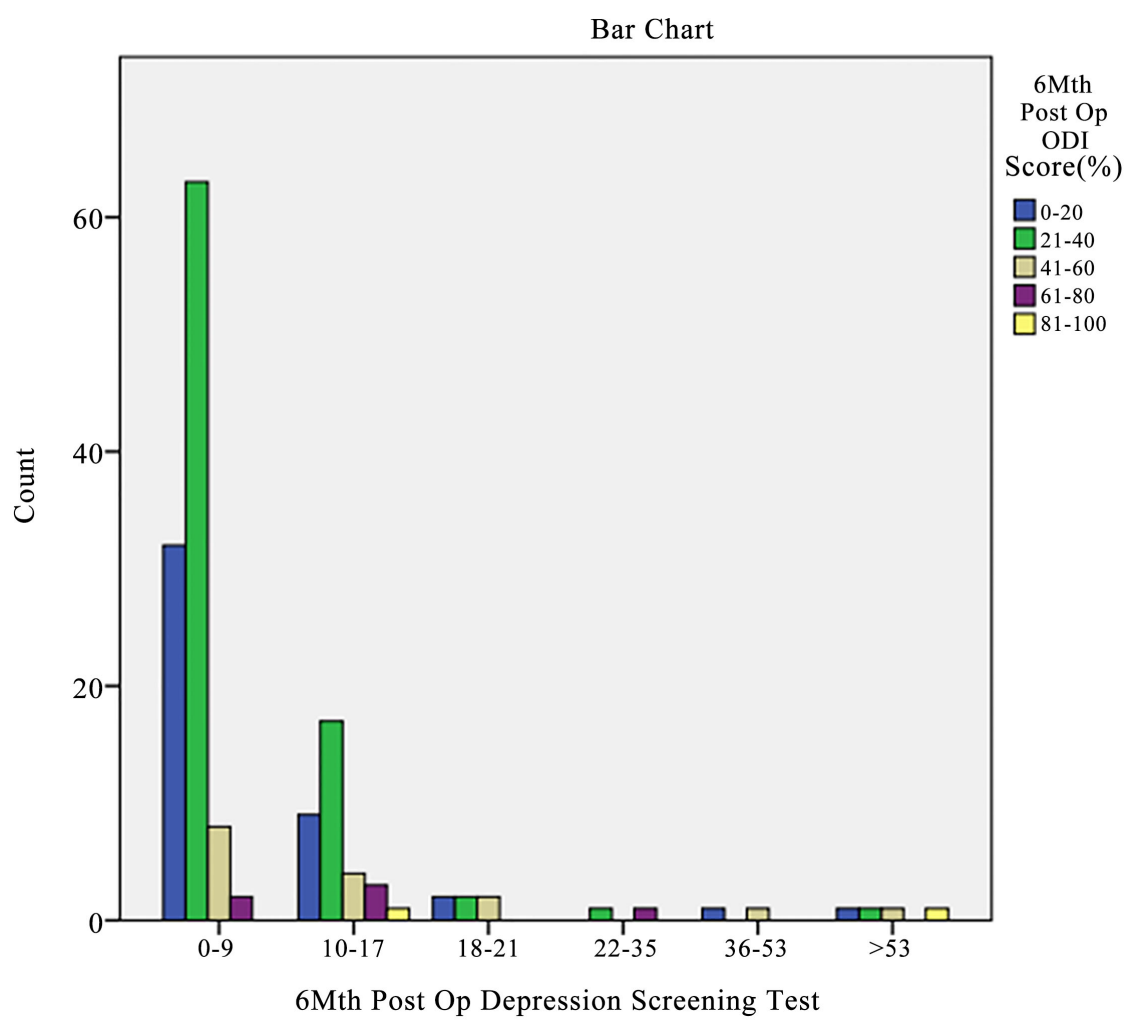

Figure 5. The relationship between level of mental Depression Score and ODI Score (\%) at 6-month after treatment. Key: Oswestry Disability Index (ODI) (\%): 0\% to 20\%: Minimal Disability; 21\% - 40\%: Moderate Disability; 41\% - 60\%: Severe Disability; 61\% 80\%: Crippled; 81\% - 100\%: Bed/Wheel Chair Bound; Depression Screening Test (Ivan Goldberg Score): 0 - 9 No Depression Likely; 10 - 17 Possibly Mildly Depressed; 18 - 21 Borderline Depression; 22 - 35 Mild-Moderate Depression; 36 - 53 Moderate-Severe Depression; $\geq 54$ Severe Depression. Comments: The relationship of the ODI scores with the level of mental Depression among the participants at 6-month follow up after treatments showed much improvement of both.

\section{Discussion}

One hundred and sixty patients were recruited into the study however 7 of them were lost to follow up while the remaining 153 participants completed the follow up periods, hence analyzed. The study involves 61 males and 92 females with ratio of 1:1.5. This ratio is similar to Igbinedion's report in Benin-City, Nigeria, where female: male of 1:1.4 was reported in a hospital based study among similar age group of patients [12].

In this study $85.6 \%$ of the patient presented at Surgical Out-Patient Department while $14.4 \%$ presented to Emergency Room which similar to finding from a study in Enugu, Nigeria where $20 \%$ of the patients presented as acute LBP to Emergency Room and the rest as chronic case in Out-patient Clinic [13]. These similar modes of presentations might be that the study was conducted in a population where patients have perception for hospital care.

In our study, we found 110 of the participants (71.9\%) presented with severe scores of ODI ( $41 \%-100 \%$ scores) but at 6 -month after treatment this reduced 
to 24 patients with severe ODI score $(41 \%$ - 100\%). This showed that many of patients presented in the late stage of this pathology. We also noted 49 (32\%) of the participants with significant mental depression symptoms at presentation while $14(9.2 \%)$ of the participants had mental depression symptoms at 6-monrth after treatments. This is the incidence of mental depression among the patients with symptomatic LDDD at presentation. This incidence of mental depression in our study is similar to the findings by Renata et al. in the study of 188 patients (48 cervical and 140 lumbar disc disease) aged 22 to 72 years in which before surgical treatment, symptoms of depression were reported in $47.3 \%$ of patients (11.7\% with cervical disc disease and 35.6\% with lumbar disc disease while 6 months after treatment the, symptoms of depression were present in $31.1 \%$ (7.5\% with cervical disc disease and 23.6\% with lumbar disc disease) [14]. The incidence of mental depression in our study is similar though smaller to the finding by Shah et al in their hospital based study in India where out of the 107 patients, 59 (55.14\%) patients were found positive for depression among people suffering from chronic low back pain (CLBP) from disc disease [2]. This level of depression is also similar (even though our finding is lower) to the report by Sharif et al. in Pakistan in a study of 140 participants with chronic low back pain from lumbar degenerative disc disease where $48.57 \%$ experienced mental depression [3]. The development of depression may be associated with chronicity of the symptoms, socioeconomic burdens, poor quality of lives and inadequacy of care especially where the spine specialists are still scanty or inadequate. The mental depression as shown by our study was not only statistically significant but also associated with symptoms severity as indicated by ODI (which measures the Level of Disability and Quality of Life among the participants). The treatment interventions showed significant improvement in both ODI and mental depression at 6-month after treatment indicating that mental depression symptoms were directly related to the symptoms severity of LDDD. In another study of similar male to female ratio (1:1.9), similar age distribution (35 to 64 years), but shorter duration of symptoms (less than 5 years), different marital status (married $78.1 \%)$ ) by Netto et al., where they noted improvement in the mean scores of the visual analogue pain scale $(p<0.001)$ and the ODI $(p<0.001)$ and improvement mental health $(p<0.001)$ [15]. In the literatures, there is associated improvement in both symptoms of LDDD and mental depression. These noted improvements may be associated with treatment of the pathology that resulted in the different symptoms especially from surgical treatment.

\section{Conclusion}

In our study, we noted the incidence of mental depression among patients with LDDD to be $32 \%$. And we also noted that there was a statistically significant relationship between symptomatic Lumbar Degenerative Disc Disease and level of mental depression with significant improvement in the level of depression at $6^{\text {th }}$ month after treatment. Hence, assessment for patients' mental depression and 
managing it is important in the management of LDDD.

\section{Recommendation}

- We recommend that all patients with symptomatic LDDD should have pre and post-treatment mental health assessment especially mental depression.

- Psychotherapy should be part of care for most patients with LDDD.

\section{Limitations of the Study}

It is a single center study done in referral orthopaedic hospital where a fraction of the people in the community presents to. A multi-centred study or community based study with a larger sample size will be more statistically significant for the conclusion. A longer follow-up period of the patients after treatment will show long term effects of the treatment types.

\section{Ethics Approval and Consent}

Approval to conduct the study from the Ethical Committee of NOHI was obtained before the study.

\section{The Researchers' Level of the Participation}

All the authors fully participated in all the stages of the study and manuscript writing.

\section{Availability of Data and Material}

This will be made available on request.

\section{Funding}

No sponsorship or funding for this study.

\section{Acknowledgements}

We acknowledged our colleague-Mental Health physicians who co-managed some of the patients with us.

\section{Conflicts of Interest}

The authors declare no conflicts of interest regarding the publication of this paper.

\section{References}

[1] Garczyk, D., Jankowski, R., Misterska, E., et al. (2013) Patient Satisfaction with Nursing after Surgery Due to Cervical or Lumbar Discopathy. Medical Science Monitor. International Medical Journal of Experimental and Clinical Research, 19, 892-902. https://doi.org/10.12659/MSM.889526

[2] Shah, S.H., Kataria, L.R. and Joshi, D. (2011) Incidence of Depression in Chronic Low-Back Pain: A Hospital Based Study. Healthline, Journal of Indian Association of Preventive and Social Medicine, 2, 35-40. 
[3] Sagheer, M.A., Khan, M.F. and Sharif, S. (2013) Association between Chronic Low Back Pain, Anxiety and Depression in Patients at a Tertiary Care Centre. J Pak Med Assoc, 63, 688-690.

[4] Bener, A., Verjee, M., Dafeeah, E.E., et al. (2013) Psychological Factors: Anxiety, Depression, and Somatization Symptoms in Low Back Pain Patients. Journal of Pain Research, 6, 95-101. https://doi.org/10.2147/JPR.S40740

[5] Middleton, K. and Fish, D.E. (2009) Lumbar Spondylosis: Clinical Presentation and Treatment Approaches. Current Reviews in Musculoskeletal Medicine, 2, 94-104. https://doi.org/10.1007/s12178-009-9051-x

[6] Merkle, M. and Wälchli, B.N. (2008) Degenerative Lumbar Spondylosis. In: Boos, N. and Aebi, M., Eds., Spinal Disorders: Fundamentals of Diagnosis and Treatment, Springer-Verlag Berlin Heidelberg, New York, 539-565.

https://doi.org/10.1007/978-3-540-69091-7_20

[7] Newman, D., O’Reilly, E.P. and Lee, S.H. (2015) Mental Health Service Users' Experiences of Mental Health Care: An Integrative Literature Review. Journal of Psychiatric and Mental Health Nursing, 22, 171-182. https://doi.org/10.1111/jpm.12202

[8] Priester, M.A., Browne, T., Iachini, A., et al. (2016) Treatment Access Barriers and Disparities among Individuals with Co-Occurring Mental Health and Substance Use Disorders: An Integrative Literature Review. Journal of Substance Abuse Treatment, 61, 47-59. https://doi.org/10.1016/j.jsat.2015.09.006

[9] Bolton, D. (2008) What Is Mental Disorder? An Essay in Philosophy, Science, and Values. Oxford University Press, Oxford. https://doi.org/10.1093/med/9780198565925.001.0001

[10] Odole, A.C., Ogunlana, M.O., Adegoke, B.O.A., Ojonima, F. and Useh, U. (2015) cNigerian Journal of Medical Rehabilitation, 18.

[11] Romano, J.M. and Turner, J.A. (1985) Chronic Pain and Depression: Does the Evidence Support a Relationship? Psychological Bulletin, 97, 18-34. https://doi.org/10.1037/0033-2909.97.1.18

[12] Igbinedion, B.E. and Akhigbe, A. (2011) Correlations of Radiographic Findings in Patients with Low Back Pain. Nigerian Medical Journal: Journal of the Nigeria Medical Association, 52, 28.

[13] Eyichukwu, O. and Ogugua, P. (2014) Epidemiology of Low Back Pain in Enugu, Nigeria. Nigerian Journal of Orthopaedics and Trauma, 11, 28-37.

[14] Jabłońska, R., Ślusarz, R., Królikowska, A., Haor, B., Antczak, A. and Szewczyk, M. (2017) Depression, Social Factors, and Pain Perception before and after Surgery for Lumbar and Cervical Degenerative Vertebral Disc Disease. Journal of Pain Research, 10, 89-99. https://doi.org/10.2147/JPR.S121328

[15] Netto, M.B., Barranco, A.B.S., de Oliveira, K.W.K. and Petronilho, F. (2018) Influence of Anxiety and Depression Symptoms on the Quality of Life in Patients Undergoing Lumbar Spine Surgery. Revista Brasileira de Ortopedia, 53, 38-44. https://doi.org/10.1016/j.rboe.2017.01.009 


\section{List of Abbreviations}

LDDD: Lumbar Degenerative Disc Disease

IVD: Inter-vertebral Disc

MRI: Magnetic Resonance Imaging

MSDs: Musculoskeletal disorders

CT scan: Computerized Tomography scan

ODI: Oswestry Disability Index

US: United State

LBP: Low Back Pain

CLBP: Chronic Low Back Pain 